\title{
Virulencia de blastosporas de Isaria fumosorosea nativas del noreste de México sobre Anastrepha ludens (Diptera: Tephritidae)
}

\author{
Virulence of blastospores of Isaria fumosorosea natives of northeastern Mexico on Anastrepha ludens (Diptera: Tephritidae)
}

\author{
FATIMA LIZETH GANDARILLA-PACHECO ${ }^{1}$, MYRIAM ELÍAS-SANTOS², MARÍA DEL SOCORRO \\ FLORES-GONZÁLEZ ${ }^{3}$, ERICK DE JESÚS DE LUNA-SANTILLANA ${ }^{4}$ e ISELA QUINTERO-ZAPATA ${ }^{5}$
}

\begin{abstract}
Resumen. Anastrepha ludens es una de las plagas que afectan severamente la producción de frutas en México y otros países neotropicales. El objetivo de este estudio fue evaluar la virulencia y el efecto en la metamorfosis de cuatro aislados nativos y una cepa de colección de Isaria fumosorosea sobre A. ludens mediante bioensayos bajo condiciones de laboratorio $\left[25 \pm 2{ }^{\circ} \mathrm{C} ; 60 \pm 5 \%(\mathrm{HR}) ; 12: 12 \mathrm{~h}(\mathrm{~L}: \mathrm{O})\right]$ al exponer larvas y pupas a una concentración de $1 \times 10^{8}$ blastosporas/ml mediante dos diferentes métodos, asperjado directo y asperjado de suelo. Los aislados HIB-23 e HIB-30 mostraron la más alta mortalidad sobre $A$. ludens mediante el método de asperjado directo sobre larvas (84 \%) mientras que el aislado HIB-19 tuvo el más alto porcentaje de interrupción de la metamorfosis (43\%) sobre $A$. ludens por el método de aspersión directa sobre pupas. Estos resultados sugieren que $A$. ludens es susceptible al ataque por I. fumosorosea y esto puede influir en la terminación de su ciclo de vida.
\end{abstract}

Palabras clave: Bioensayos, larvas, metamorfosis, mosca mexicana de la fruta, pupas.

\begin{abstract}
Anastrepha ludens is one of the pests that seriously affects fruit production in Mexico and other Neotropical countries. The aim of this study was to evaluate the virulence and effects of four native isolates and a collected strain of Isaria fumosorosea on the metamorphosis of $A$. ludens through the use of bioassays under laboratory conditions $\left[25 \pm 2{ }^{\circ} \mathrm{C} ; 60 \pm 5 \%(\mathrm{HR}) ; 12: 12 \mathrm{~h}(\mathrm{~L}: \mathrm{O})\right]$. Larvae and pupae were exposed to $1 \times 10^{8}$ blastospores/ ml using two different methods: direct spraying and spraying of soil. The isolates HIB-23 and HIB-30 showed the highest mortality on $A$. ludens when applied using the direct spraying method on larvae (84\%), while the isolate HIB-19 showed the highest percentage of interruption of the metamorphosis ( $43 \%$ ) of $A$. ludens when applied through the method of direct spraying on pupae. The results show that $A$. ludens is susceptible to the attack of I. fumosorosea, which causes the disruption of its life cycle.
\end{abstract}

Key words: Bioassays, larvae, metamorphosis, Mexican fruit fly, pupae.

\section{Introducción}

La mosca mexicana de la fruta, Anastrepha ludens (Loew, 1873) (Diptera: Tephritidae) es una plaga nativa del noreste de México y está en América Central hasta Costa Rica y con amplia distribución en las regiones citrícolas de la costa oeste de México y Texas en los Estados Unidos en donde se llevan a cabo actividades para su detección, muestreo y erradicación (Weems et al. 2001). A. ludens es considerada una especie de importancia económica por el impacto y la severidad del daño que causa en los frutos; además se encuentra catalogada como una plaga cuarentenaria y polífaga ya que sus hospederos incluyen varias familias de plantas. En México entre sus huéspedes naturales están los frutos de mango (Mangifera indica), diversos Citrus spp. (Rutaceae) y, en ocasiones, infesta frutos de melocotón (Prunus persicae) y algunas frutas de la familia Myrtaceae (Hernández-Ortiz 2007).

Anastrepha ludens causa daño directo cuando deposita sus huevos en el fruto; una vez que emergen las larvas se alimentan del fruto, causando su caída y la contaminación por patógenos; pérdidas del 10 al $25 \%$ de la producción de mango, guayaba y cítricos se pueden presentar debido a las actividades de alimentación de $A$. ludens. En suma, los daños directos causan pérdidas en la producción de los frutos e incrementan los costos; por otra parte, los indirectos incluyen las restricciones relacionadas con la exportación y en el mercado doméstico se añaden a la exportación y comercialización interna, la construcción y mantenimiento de las instalaciones para el tratamiento de las frutas y los programas de erradicación (Stibick 2004). La infestación por moscas de la fruta es uno de los problemas críticos en el mercado internacional de cuarentena de frutas; cuando los países importadores exigen tratamientos de salud post-cosecha, los productores exportadores deben aplicar los tratamientos aprobados para eliminar las plagas (Gazit et al. 2004).

Los programas de manejo de esta plaga llevados a cabo en países como México, se basan en un sistema de manejo integrado de plagas (MIP) que comprende acciones de monitoreo (trampeo y muestreo de fruta) y un control con pulverización cebo específico, actividades culturales, la liberación de

\footnotetext{
${ }^{1}$ Posdoctorado en ciencias, Universidad Autónoma de Nuevo León, Facultad de Ciencias Biológicas, Instituto de Biotecnología, Av. Pedro de Alba s/n cruz con Av. Manuel L. Barragán, Ciudad Universitaria, C.P. 66455, San Nicolás de los Garza, Nuevo León, México. ${ }^{2}$ Doctor en ciencias, Universidad Autónoma de Nuevo León, Facultad de Ciencias Biológicas, Instituto de Biotecnología, Av. Pedro de Alba s/n cruz con Av. Manuel L. Barragán, Ciudad Universitaria, C.P. 66455 , San Nicolás de los Garza, Nuevo León, México. ${ }^{3}$ Doctor en ciencias, Universidad Autónoma de Nuevo León, Facultad de Ciencias Biológicas, Instituto de Biotecnología, Av. Pedro de Alba s/n cruz con Av. Manuel L. Barragán, Ciudad Universitaria, C.P. 66455, San Nicolás de los Garza, Nuevo León, México. ${ }^{4}$ Doctor en ciencias, Instituto Politécnico Nacional, Centro de Biotecnología Genómica, Laboratorio de Medicina de Conservación, Blvd. del Maestro s/n esq. Elías Piña, Col. Narciso Mendoza, C.P. 88710, Reynosa, Tamaulipas, México. ${ }^{5}$ Doctor en ciencias, Universidad Autónoma de Nuevo León, Facultad de Ciencias Biológicas, Instituto de Biotecnología, Av. Pedro de Alba s/n cruz con Av. Manuel L. Barragán, Ciudad Universitaria, C.P. 66455, San Nicolás de los Garza, Nuevo León, México. Autor de correspondencia: Isela Quintero-Zapata, Universidad Autónoma de Nuevo León, Facultad de Ciencias Biológicas, Instituto de Biotecnología, Av. Pedro de Alba s/n cruz con Av. Manuel L. Barragán, Ciudad Universitaria, C.P. 66455, San Nicolás de los Garza, Nuevo León, México, isela.quinterozp@uanl.edu.mx.
} 
enemigos naturales como Diachasmimorpha longicaudata (Ashmead, 1905) (Hymenoptera: Braconidae) y moscas estériles. La aplicación coordinada de estas actividades tiene como objetivo lograr el establecimiento de zonas libres y de baja prevalencia de la plaga para permitir la producción de plantas y frutas de óptima calidad para facilitar el acceso a los mercados nacionales e internacionales.

Sin embargo, es necesario desarrollar alternativas que puedan considerarse complementarias, haciendo hincapié en aquellas que tienen un menor impacto en el medio ambiente y una mayor aceptación de los productores en los sistemas de agricultura orgánica (Muñoz et al. 2009). En este sentido, los hongos entomopatógenos ofrecen un gran potencial, ya que se ha demostrado su eficacia como agentes de control de diferentes plagas agrícolas (Lecuona et al. 1996) y porque, además, tienen un mecanismo de invasión único que les permite pasar a través de la cutícula, dirigirse a diferentes órganos dentro del insecto incluyendo la pared del aparato digestivo, lo que los hace excelentes agentes de control biológico ya que actúan como insecticidas de contacto (Charnley y Collins 2007). En el caso de moscas de la fruta se ha demostrado que algunas cepas de estos hongos causan una alta mortalidad en adultos (Castillo et al. 2000; De la Rosa et al. 2002).

La etapa conidial de varias cepas de Beauveria bassiana (Bals.) Vuillemin y Metarhizium anisopliae (Metsch.) Sorokin, de distintas regiones geográficas han sido evaluadas bajo condiciones de laboratorio contra diferentes etapas biológicas de varias especies de moscas de la fruta (García et al. 1984; Espín-García et al. 1989; Castillo et al. 2000; De la Rosa et al. 2002; Ekesi et al. 2005; Yee y Lacey 2005), pero poco se conoce sobre otras especies de entomopatógenos, así como otros propágulos que podrían ayudar a incrementar las tasas de mortalidad. El objetivo de este estudio fue evaluar la patogenicidad de blastosporas de aislados nativos de Isaria fumosorosea (= Paecilomyces fumosoroseus) procedentes del noreste de México sobre larvas y pupas de A. ludens, así como el efecto en su ciclo de vida.

\section{Materiales y métodos}

Cría de $\boldsymbol{A}$. ludens. La cría masiva de larvas y pupas de $A$. ludens, se desarrolló en dieta artificial en condiciones de laboratorio a $\left[25 \pm 2{ }^{\circ} \mathrm{C} ; 60 \pm 5 \%\right.$ (HR); 12:12 h (L: O)] en la unidad de cría masiva de insectos del Instituto de Biotecnología, (IB-FCB-UANL), San Nicolás de los Garza, Nuevo León, México.

Aislamientos de I. fumosorosea. Los aislamientos de I. fumosorosea se obtuvieron a partir de suelos de la región citrícola del estado de Nuevo León, México (Tabla 1). El método empleado para el aislamiento de los hongos fue descrito por Galán-Franco et al. (2011). Los cultivos fueron depositados en la colección de laboratorio L-6 del Instituto de Biotecnología, (IB-FCB-UANL). Para su conservación se utilizaron viales criogénicos con $1 \mathrm{ml}$ de glicerol a $10 \% \mathrm{v} / \mathrm{v}$, almacenado en congelación a $-80^{\circ} \mathrm{C}$. Además, en el estudio se incluyó la cepa Pfr-612.

Medio de cultivo líquido para la producción de blastosporas. La composición del medio basal para la producción de blastosporas en cultivo sumergido fueron los siguientes: $\mathrm{KH}_{2} \mathrm{PO}_{4}, 2.0 \mathrm{~g} ; \mathrm{CaCl}_{2} 2 \mathrm{H}_{2} \mathrm{O}, 0.4 \mathrm{~g} ; \mathrm{MgSO}_{4} 7 \mathrm{H}_{2} \mathrm{O}, 0.3 \mathrm{~g}$; Co$\mathrm{Cl}_{2} 6 \mathrm{H}_{2} \mathrm{O}, 37 \mathrm{mg} ; \mathrm{FeSO}_{4} 7 \mathrm{H}_{2} \mathrm{O}, 50 \mathrm{mg} ; \mathrm{MnSO}_{4} \mathrm{H}_{2} \mathrm{O}, 16 \mathrm{mg}$;
Tabla 1. Localización de aislados de I. fumosorosea en suelos donde se cultivan cítricos en diferentes localidades del estado de Nuevo León, México.

\begin{tabular}{|c|c|c|c|}
\hline Clave & $\begin{array}{l}\text { Localidad } \\
\text { de colecta }\end{array}$ & Localización geográfica & $\begin{array}{c}\text { Elevación } \\
(\mathrm{msnm})\end{array}$ \\
\hline HIB-19 & Linares & $25^{\circ} 09^{\prime} 13^{\prime \prime} \mathrm{N} 99^{\circ} 51^{\prime} 10^{\prime \prime O}$ & 350 \\
\hline HIB-23 & Montemorelos & $25^{\circ} 10^{\prime} 03^{\prime \prime} \mathrm{N} 99^{\circ} 51^{\prime} 10^{\prime \prime O}$ & 430 \\
\hline HIB-29 & Montemorelos & $25^{\circ} 18^{\prime} 12^{\prime \prime} \mathrm{N} 99^{\circ} 53^{\prime} 13^{\prime \prime O}$ & 430 \\
\hline HIB-30 & Montemorelos & $25^{\circ} 10^{\prime} 03$ ”N 9957'11"O & 430 \\
\hline
\end{tabular}

$\mathrm{ZnSO}_{4} 7 \mathrm{H}_{2} \mathrm{O}, 14 \mathrm{mg}$. Al medio basal se agregaron glucosa (Jalmek, San Nicolás de los Garza, N.L., México) 80 y 25 g/l de casaminoácidos (BD, N.J., EE. UU.). La solución de glucosa fue esterilizada por separado antes de agregarse al medio basal (Jackson et al. 1997). Para la producción de blastosporas todos los hongos fueron cultivados durante 14 a 21 días en agar papa dextrosa (DIBICO, México, D.F.) a $25 \pm$ $2{ }^{\circ} \mathrm{C}$. Después se prepararon suspensiones de $5 \times 10^{5}$ conidios/ $\mathrm{ml}$ con agua destilada estéril para inocular el cultivo líquido (sumergido). Se usaron matraces bafleados (Pyrex, N.Y., EE. UU.) de $250 \mathrm{ml}$ con $100 \mathrm{ml}$ de medio, se incubaron a 28 ${ }^{\circ} \mathrm{C}$ y $300 \mathrm{rpm}$ en un incubador con agitación orbital (New Brunswick Scientific, N.J., EE. UU.) (Jackson et al. 1997) durante 72 horas. Una vez concluida la fase de producción la concentración se ajustó a $1 \times 10^{8}$ blastosporas $/ \mathrm{ml}$ utilizando una cámara de Neubauer para ser utilizadas inmediatamente en los bioensayos.

Bioensayos. Para los bioensayos se utilizaron muestras de suelo agrícola, tamizados y esterilizados en recipientes de vidrio durante 30 minutos a 15 libras de presión. Después se colocaron $200 \mathrm{~g}$ del suelo esterilizado en recipientes de plástico donde el suelo se humedeció con agua bidestilada hasta que el suelo estaba completamente húmedo. Para la inoculación de larvas y pupas se utilizaron dos métodos: asperjado directo y asperjado de suelo. Para el primero se rocío directamente con un atomizador manual en cinco ocasiones las pupas y larvas con la solución de blastosporas de cada uno de los aislados $\left(1 \times 10^{8}\right.$ blastosporas $\left./ \mathrm{ml}\right)$, mientras que para el método de asperjado de suelo, el cual fue previamente esterilizado y humedecido, éste se roció con un atomizador manual en cinco ocasiones con la solución de blastosporas de cada uno de los aislados utilizados $\left(1 \times 10^{8}\right.$ blastosporas $\left./ \mathrm{ml}\right)$. A continuación para ambos métodos, larvas y pupas se colocaron respectivamente en los contenedores con suelo estéril y luego se cerraron herméticamente y se pusieron en posición invertida. Para ambos métodos se realizaron tres repeticiones por tratamiento, incluyendo un testigo con agua bidestilada y uno no tratado. Posteriormente, cada contenedor fue incubado a [26 $\pm 2{ }^{\circ} \mathrm{C} ; 60 \pm 5 \%$ (HR); 12:12 h (L: O)] durante siete días. Al séptimo día se registró el porcentaje de larvas y pupas infectadas para cada uno de los aislados utilizados. Las larvas y pupas infectadas se colocaron en cajas de Petri de $60 \times 15 \mathrm{~mm}$ y se incubaron a $26 \pm 2{ }^{\circ} \mathrm{C}$ y $70 \pm 5 \%$ H.R durante siete días para estimular el desarrollo de micosis y verificar la esporulación característica de I. fumosorosea. Las larvas o pupas que no mostraron signos de infección se incubaron bajo las condiciones iniciales $\left[26 \pm 2{ }^{\circ} \mathrm{C} ; 60 \pm 5 \%\right.$ (HR); 12:12 h (L: O)] y se monitorearon hasta completar su ciclo de vida. Las larvas y pupas que se consideraron infectadas son las que mostraron signos o síntomas de infección 
por entomopatógenos como esporulación y cambios de color o tamaño.

Análisis de datos. El experimento se realizó, al menos dos ocasiones, bajo un diseño completamente al azar con cinco aislados fúngicos, con tres repeticiones por aislado, con 15 larvas o pupas por repetición. Los resultados se expresaron como porcentaje con su respectivo intervalo de confianza al $95 \%$. Las tasas de mortalidad se corrigieron mediante la fórmula de Schneider-Orelli. La normalidad de los datos se verificó con las pruebas de Shapiro-Wilk y de T $(\mathrm{P}<0,05)$ para comparar las medias. Los datos se analizaron utilizando el programa SPSS ${ }^{\circledR}$ IBM $^{\circledR}$ v.19 Inc., N.Y.

\section{Resultados y discusión}

Los resultados mostraron que las tasas de mortalidad para el método de aspersión directa sobre larvas fue 50-84 \%, mientras que para el método de aspersión directa al suelo con larvas fue de 41-75\% y de acuerdo a los resultados del análisis estadístico no se encontró diferencia significativa entre los dos métodos de bioensayo $(\mathrm{t}=1,23$; $\mathrm{df}=8 ; \mathrm{P}=0,239)$. Respecto a la mortalidad de pupas se registraron valores de 5-20 \% para el método de aspersión directa a pupas, mientras que para el método de aspersión directa a suelo con pupas la mortalidad fue de $17-50 \%$ y no se encontró diferencia significativa entre los dos métodos de bioensayo $(\mathrm{t}=0,68 ; \mathrm{df}=8 ; \mathrm{P}=$ $0,514)$, mientras que si detectó diferencia significativa entre los aislados evaluados sobre $A$. ludens $(\mathrm{t}=5,31 ; \mathrm{df}=19 ; \mathrm{P}=$ 0,0001). Los aislados HIB-23 e HIB-30 mostraron la más alta mortalidad sobre $A$. ludens por el método de aspersión directa sobre larvas (Tabla 2).

La interrupción de la metamorfosis se registró de 20-43 \% para el método de aspersión directa sobre pupas, mientras que para el de aspersión directa sobre suelo con pupas se obtuvieron valores de $33 \%$ sin presentar diferencia significativa entre ambos métodos $(\mathrm{t}=2,01 ; \mathrm{df}=8 ; \mathrm{P}=0,079)$, a diferencia entre los aislados evaluados $(\mathrm{t}=9,40 ; \mathrm{df}=19 ; \mathrm{P}=$ 0,0001). Los aislados HIB-19 e HIB-23 mostraron el porcentaje más alto de interrupción de la metamorfosis de $A$. ludens por el método de aspersión directa sobre pupas mientras que no existió para los métodos de aspersión directa sobre larvas y sobre suelo con larvas (Tabla 3 ). En relación a la terminación del ciclo de vida el porcentaje más alto de supervivencia de $A$. ludens se registró con el método de aspersión directa sobre suelo con pupas con el aislado HIB-30 (67 \%), mien- tras que el porcentaje más bajo de supervivencia fue por los de aspersión directa sobre larvas y pupas (13\%); de acuerdo al análisis estadístico no existió diferencia significativa entre los métodos de bioensayo respecto a la terminación del ciclo de vida de $A$. ludens (aspersión directa sobre larvas y aspersión directa sobre suelo con larvas $(\mathrm{t}=1,23 ; \mathrm{df}=8 ; \mathrm{P}$ $=0,253)$, y aspersión directa sobre pupas y aspersión directa sobre suelo con pupas $(\mathrm{t}=1,00 ; \mathrm{df}=8 ; \mathrm{P}=0,344)$, mientras que si existió diferencia significativa entre los hongos evaluados para la supervivencia y terminación del ciclo de vida de $A$. ludens ( $\mathrm{t}=9,40 ; \mathrm{df}=19 ; \mathrm{P}=0,0001)$ (Tabla 4).

En los trabajos que utilizan hongos entomopatógenos, la mayoría son a nivel laboratorio, para el control de $A$. ludens difieren notablemente en los métodos empleados para la evaluación así como en los géneros de hongos y concentración de esporas como inóculo, sin embargo, coinciden en el uso de conidios como propágulo para la evaluación. En el presente estudio se utilizaron blastosporas de $I$. fumosorosea sobre larvas y pupas de $A$. ludens como inóculo mediante dos métodos de bioensayo diferentes obteniendo mortalidades superiores al $80 \%$. En un estudio previo, con conidios de I. fumosorosea, provenientes de la misma colección de hongos utilizados en el presente estudio, se obtuvieron mortalidades superiores al $62 \%$ mediante el sumergido de pupas y del $57 \%$ mediante el método de suelo asperjado que contenía larvas de $A$. ludens (Gandarilla-Pacheco et al. 2012).

En otro estudio, De la Rosa et al. (2002) reportaron mortalidades mínimas para $A$. ludens con un $8,2 \%$ en larvas y de cero en pupas, mientras que en adultos las mortalidades alcanzaron de 98-100\% con cepas de B. bassiana a una concentración de 1,0 - 1,6 × $10^{8}$ conidios $/ \mathrm{ml}$. Así mismo, Lezama-Gutiérrez et al. (2000) con aislados de Metarhizium anisopliae reportaron mortalidades de 98,75\% sobre larvas de $A$. ludens a una concentración de $1 \times 10^{8}$ conidios $/ \mathrm{ml}$. Estos resultados muestran una alta variabilidad en las tasas de mortalidad obtenidas con las diferentes especies de hongos probadas. En la literatura son numerosos los reportes que indican que existen diferencias en la susceptibilidad de un insecto en particular a determinado hongo entomopatógeno y estas diferencias entre poblaciones de insectos se deben a asociaciones prolongadas del huésped con el patógeno causando un proceso de selección natural (Lecuona et al. 1996). Factores endógenos y exógenos están relacionados con la tolerancia o susceptibilidad de un huésped a un patógeno en particular pero el efecto no está completamente claro, porque actúan en una asociación entre el insecto y el patógeno, por

Tabla 2. Mortalidad (IC $95 \%$ )* de larvas y pupas de Anastrepha ludens tratadas con aislados nativos de hongos entomopatógenos mediante dos diferentes métodos de aplicación bajo condiciones de laboratorio $\left[26 \pm 2{ }^{\circ} \mathrm{C} ; 60 \pm 5 \%(\mathrm{HR}) ; 12: 12\right.$ h (L: O)].

\begin{tabular}{lccccc}
\hline \multirow{2}{*}{ Aislado } & \multicolumn{2}{c}{ Larvas } & & \multicolumn{2}{c}{ Pupas } \\
\cline { 2 - 3 } \cline { 5 - 6 } & Asperjado directo & Asperjado de suelo & & Asperjado directo & Asperjado de suelo \\
\hline HIB-19 & $75(74,90-75,09)$ & $41(40,88-41,11)$ & & $20(19,91-20,09)$ & $50(49,88-50,11)$ \\
HIB-23 & $84(83,91-84,08)$ & $75(74,90-75,09)$ & & $10(9,93-10,06)$ & $17(16,92-17,08)$ \\
HIB-29 & $50(49,88-50,11)$ & $58(57,88-58,11)$ & & 0 & 0 \\
HIB-30 & $84(83,91-84,08)$ & $58(57,88-58,11)$ & & $5(4,96-5,04)$ & 0 \\
Pfr-612 & $50(49,88-50,11)$ & $50(49,88-50,11)$ & & 0 & $3(2,97-3,03)$ \\
Media \pm EE & $68,60 \pm 7,76$ & $56,40 \pm 5,60$ & & $7,00 \pm 3,74$ & $14,00 \pm 9,53$ \\
\hline
\end{tabular}

* Mortalidad promedio es reportada en porcentaje (\%). Testigo con agua bidestilada y testigo no tratado: $20 \%$. 
Tabla 3. Interrupción de la metamorfosis promedio* (IM) (IC $95 \%$ ) en pupas de Anastrepha ludens tratadas con aislados nativos de hongos entomopatógenos mediante dos diferentes métodos de aplicación bajo condiciones de laboratorio $\left[26 \pm 2{ }^{\circ} \mathrm{C} ; 60 \pm 5 \%(\mathrm{HR}) ; 12: 12 \mathrm{~h}(\mathrm{~L}: \mathrm{O})\right]$.

\begin{tabular}{lcc}
\hline \multicolumn{1}{c}{ Aislado } & Asperjado directo & Asperjado de suelo \\
\hline HIB-19 & $43(42,89-43,11)$ & $20(19,91-20,09)$ \\
HIB-23 & $37(36,90-37,10)$ & $33(32,90-33,10)$ \\
HIB-29 & $20(19,91-20,09)$ & $17(16,92-17,08)$ \\
HIB-30 & $33(32,90-33,10)$ & $17(16,92-17,08)$ \\
Pfr-612 & $27(26,9-27,10)$ & $23(22,91-23,09)$ \\
Media \pm EE & $32,00 \pm 3,97$ & $22,00 \pm 2,96$ \\
\hline
\end{tabular}

* Interrupción de la metamorfosis promedio (IM) es reportada en porcentaje (\%).

lo que los factores endógenos (fisiológicos y genéticos) se asocian e influyen en la susceptibilidad del insecto huésped; sin embargo, éstos pueden ser modificados por factores exógenos tales como la temperatura y la alimentación de insecto huésped (Watanabe 1987).

Otros registros involucran otras especies de moscas de la fruta y hongos entomopatógenos con resultados similares. Quesada-Moraga et al. (2006) reportaron mortalidades de 95-100 \% sobre adultos de la mosca mediterránea de la fruta Ceratitis capitata (Wiedemann, 1824) (Diptera: Tephritidae), y de 94,5 \% sobre pupas utilizando conidios de B. bassiana y M. anisopliae. Hernández Díaz-Ordaz et al. (2010) reportaron mortalidades de hasta 99,8 \% sobre adultos de Anastrepha obliqua (Macquart, 1835) (Diptera: Tephritidae) con cepas de B. bassiana y M. anisopliae. Variaciones en la mortalidad pueden depender de las cepas utilizadas, origen del hospedero, y modo de infección; es recomendable que antes de comenzar la investigación sobre el uso de entomopatógenos, las mejores cepas para el control biológico del insecto objeto de estudio sean seleccionadas por medio de pruebas básicas de patogenicidad (Roberts 1989).

En el presente estudio las mortalidades más altas se observaron por el método de aspersión directa a larvas, lo cual es lo esperado si se tiene en cuenta que con los otros métodos los propágulos tenían una menor posibilidad de contacto con las larvas y pupas, debido a que el área de inoculación es mayor en el caso de suelo asperjado y en el de la aspersión directa a pupas éstas poseen una cutícula gruesa y completamente esclerotizada, y una cápsula pupal dura y engrosada con una superficie escamosa que podría explicar las bajas tasas de mortalidad con este método (De la Rosa et al. 2002). Otro factor en el caso de las pupas, es la ausencia de movimiento para ponerse en contacto con el suelo, que redujo la posibilidad de ser infectadas con propágulos viables que puedan estar en el suelo en el momento de la inoculación, esta situación se reduce en el caso de las larvas debido a su capacidad de moverse en el sustrato. Con respecto al tipo de propágulo utilizado, en la mayoría de los trabajos mencionados son los conidios de diferentes especies de hongos entomopatógenos más comúnmente ensayados en estrategias de control biológico debido a su conocida hidrofobicidad y resistencia a la desecación lo cual puede proveerles algunas ventajas (Jackson et al. 1997).

El hongo, sus diversos aislados y el tipo de propágulo utilizado es decir blastosporas en comparación con conidios, pueden ser responsables de la eficacia (Lacey et al. 2008).

Lacey et al. (1999) reportaron que blastosporas recién producidas fueron significativamente más infecciosas para ninfas de Bemisia argentifolii (Bellows \& Perring, 1994) (Hemiptera: Aleyrodidae) que conidios del mismo aislado de I. fumosorosea. Lane et al. (1991) sugirieron que la naturaleza hidrofóbica de conidios de $B$. bassiana aumenta la adherencia de estos propágulos a la cutícula del saltahojas verde, Nephotettix virescens (Distant, 1908) (Hemiptera: Cicadellidae), mejorando significativamente la eficacia de biocontrol de conidios en comparación con blastosporas de B. bassiana. Es posible que el problema de adherencia es menos importante cuando se trata con ninfas sésiles como $B$. argentifolii en lugar de insectos móviles. También es probable que la tasa de germinación rápida de las blastosporas en comparación con los conidios sea responsable de la mejora en la eficacia de estos propágulos infectando y matando ninfas de $B$. argentifolii (Jackson et al. 1997).

Esto podría explicar el éxito obtenido con blastosporas en el presente estudio para infectar las larvas y pupas de $A$. ludens en comparación con el estudio realizado por Gandarilla-Pacheco et al. (2012) con conidios de otras aislados de la misma colección. Además, las blastosporas utilizadas se produjeron de acuerdo con la metodología propuesta por Jackson et al. (1997) que se basa en el cultivo líquido de blastosporas P. fumosoroseus estables y tolerantes a la desecación.

Respecto a la interrupción de la metamorfosis de $A$. ludens se registraron valores de hasta $43 \%$ por el método de aspersión directa a pupas y $33 \%$ para el método de aspersión a suelo. Estos resultados son importantes porque al retrasar el desarrollo se incapacita al insecto para alcanzar el esta-

Tabla 4. Sobrevivencia de larvas y pupas (S) (IC $95 \%$ ) de Anastrepha ludens tratadas con aislados nativos de hongos entomopatógenos mediante dos diferentes métodos de aplicación bajo condiciones de laboratorio $\left[26 \pm 2{ }^{\circ} \mathrm{C} ; 60 \pm 5 \%\right.$ (HR); 12:12 h (L: O)].

\begin{tabular}{lccccc}
\hline \multirow{2}{*}{ Aislado } & \multicolumn{2}{c}{ Larvas } & & \multicolumn{2}{c}{ Pupas } \\
\cline { 2 - 3 } \cline { 5 - 6 } & Asperjado directo & Asperjado de suelo & & Asperjado directo & Asperjado de suelo \\
\hline HIB-19 & $20(19,91-20,09)$ & $47(46,89-47,11)$ & & $13(12,93-13,07)$ & $20(19,91-20,09)$ \\
HIB-23 & $13(12,93-13,07)$ & $20(19,91-20,09)$ & & $26(25,91-26,09)$ & $33(32,90-33,10)$ \\
HIB-29 & $40(39,89-40,11)$ & $33(32,90-33,10)$ & & $60(59,89-60,11)$ & $66(65,90-66,10)$ \\
HIB-30 & $13(12,93-13,07)$ & $33(32,90-33,10)$ & & $33(32,90-33,10)$ & $67(66,90-67,10)$ \\
Pfr-612 & $40(39,89-40,11)$ & $40(39,89-40,11)$ & & $46(45,89-46,11)$ & $54(53,89-54,11)$ \\
Media \pm EE & $25,20 \pm 6,17$ & $34,60 \pm 4,47$ & & $35,60 \pm 8,10$ & $48,00 \pm 9,30$ \\
\hline
\end{tabular}

* Sobrevivencia promedio (S) es reportada en porcentaje (\%). 
do adulto y reproducirse y, por lo tanto, el seguimiento de este parámetro es de vital importancia. Pocos estudios explican a fondo la interrupción de la metamorfosis debido a la acción de los hongos entomopatógenos, específicamente por I. fumosorosea. Sin embargo, Azevedo (1998) menciona que la acción de las toxinas tales como las producidas por $B$. bassiana (beauvericina) y destruxinas producidas por M. anisopliae puede causar alteraciones en los mecanismos de metamorfosis y de defensa. En contraste, Zimmermann (2008) considera que con B. bassiana y M. anisopliae, hay información limitada sobre los metabolitos y toxinas producidas por las especies de Isaria. La beauvericina también se ha aislado a partir de diferentes especies de Fusarium y Paecilomyces (Gupta y Krasnoff 1991).

Jegorov et al. (1994) mencionan que dos beauverolides ( $\mathrm{L}$ y La) fueron identificados a partir de I. fumosorosea. La beauvericina es un ciclopéptido y su biosíntesis implica una enzima multifuncional llamada eniantinasintetasa cuya expresión es constitutiva (Billich y Zocher 1988). Se sabe que es un agente ionóforo capaz de acomplejar iones de $\mathrm{Na}^{+} \mathrm{y} \mathrm{K}^{+}$ que permiten el aumento de la permeabilidad de la membrana e inducen la deshidratación del tejido por la pérdida de fluido de la célula. Esto se supone que es la principal causa de muerte del insecto, así como los cambios causados en el núcleo de la célula (Ovchninnikov et al. 1971). Además, pueden causar cambios en los procesos de muda, metamorfosis y fertilidad (Ferron 1978) como se menciona en Castillo et al. (2000) con I. fumosorosea (cepa CG-260) que resultó eficaz contra los adultos de $C$. capitata con valores de $\mathrm{DL}_{50}$ de 10 días de 6,1 $\times 10^{3}$ conidios por mosca. La cepa CECT 2705 causó reducciones en la fecundidad del orden de $65 \%$ a $1 \times 10^{6}$ conidios por mosca. Por otra parte, Roberts (1981) menciona al ácido piridina 2,6-dicarboxílico (ácido dipicolínico), aislado a partir de Isaria farinosa e I. fumosorosea, mostró una actividad insecticida contra ninfas de tercer estadio de Bemisia tabaci tipo B (=B. argentifolii) (Asaff et al. 2005). La secreción de uno o más de estos metabolitos por los aislados utilizados en este estudio acerca de $A$. ludens puede haber causado la mortalidad o interrupción en la metamorfosis, sin embargo, se requieren métodos analíticos para comprobar el papel que pueden desempeñar en el proceso de infección estos metabolitos.

\section{Conclusiones}

El uso de blastosporas de aislados nativos de I. fumosorosea mostró efectividad de hasta el $84 \%$ mediante el asperjado directo sobre larvas, así como un efecto significativo en la interrupción de la metamorfosis de $A$. ludens. Estos resultados indican que la mosca mexicana de la fruta es propensa a la infección por I. fumosorosea lo cual puede influir en la terminación de su ciclo de vida y es promisorio en mermar la capacidad reproductiva del insecto. Lo anterior en combinación con otras estrategias ayudaría a disminuir la población hasta niveles sub económicos. Por otra parte, contar con aislamientos de hongos procedentes de la región y adaptados a las condiciones climáticas de la misma puede influir de manera positiva en su capacidad como patógeno sobre el insecto de interés. Este trabajo propone bases para continuar con investigaciones que permitan establecer alternativas viables para el manejo de $A$. ludens, haciendo uso de estrategias de control biológico en un marco de manejo integral de la plaga.

\section{Agradecimientos}

Los autores agradecen al proyecto PAICYT CN 1008-11.

\section{Literatura citada}

ASAFF, A.; CERDA-GARCÍA-ROJAS, C.; DE LA TORRE, M. 2005. Isolation of dipicolinic acid as an insecticidal toxin from Paecilomyces fumosoroseus. Applied Microbiology and Biotechnology 68 (4): 542-547.

AZEVEDO, J. L. 1998. Controle microbiano de insetos pragas e seu melhoramento genético. pp. 69-93. En: de Melo, I. S.; de Azevedo, J.L. (Eds.). Controle biológico. Volume 1. Ministerio da Agriculturae do Abastecimiento, Empresa Brasileira de Pesquisa Agropecuaria (EMBRAPA), Piracicaba, Sao Paulo, Brasil, $264 \mathrm{p}$.

BILLICH, A.; ZOCHER, R. 1988.Constitutive expression of enniatin synthetase during fermentative growth of Fusarium scirpi. Applied and Environmental Microbiology 54 (10): 2504-2509.

CASTILLO, M. A.; MOYA, P.; HERNÁNDEZ, E.; PRIMO-YÚFERA, E. 2000. Susceptibility of Ceratitis capitata Wiedemann (Diptera: Tephritidae) to entomopathogenic fungi and their extracts. Biological Control 19 (3): 274-282.

CHARNLEY, A. K.; COLLINS, S. A. 2007. Entomopathogenic fungi and their role in pest control. pp. 159-187. En: Kubicek, C.P.; Druzhinina, I.S. (Eds.). Environmental and Microbial Relationship. The Mycota IV. Springer-Verlag, Berlín. 350 p.

DE LA ROSA, W.; LÓPEZ, F. L.; LIEDO, P. 2002. Beauveria bassiana as a pathogen of the Mexican fruit fly (Diptera: Tephritidae) under laboratory conditions. Journal of Economic Entomology 95 (1): 36-43.

EKESI, S.; MANIANIA, N. K.; MOHAMED, S. A.; LUXS. A. 2005. Effect of soil application of Metarhizium anisopliae on African tephritid fruit flies and their associated endoparasitoids. Biological Control 35 (1): 83-91.

ESPÍN-GARCÍA, G. A. T.; SOUZA, H. M. L.; MESSIAS, C. L.; PIEDRABUENA, A. E. 1989. Patogenicidade de Metarhizium anisopliae nas diferentes fases de desenvolvimiento de Ceratitis capitata (Wied.) (Diptera: Tephritidae). Revista Brasileira de Entomologia 33 (1): 17-23.

FERRON, P. 1978. Biological control of insect pests by entomogenous fungi. Annual Review of Entomology 23: 409-442.

GALÁN-FRANCO, L. A.; MORALES-LOREDO, A.; ÁLVAREZOJEDA, G.; LÓPEZ-ARROYO, J. I.; ARÉVALO-NIÑO, K.; SANDOVAL-CORONADO, C.; QUINTERO-ZAPATA, I.2011. Isolation and characterization of entomopathogenic fungi obtained from citrus-growing areas of Mexico. Southwestern Entomologist 36 (4): 443-449.

GANDARILLA-PACHECO, F. L.; NAVA- GONZÁLEZ, H. D.; ARÉVALO-NIÑO, K.; GALÁN WONG, L. J.; ELÍAS-SANTOS, M.; QUINTERO-ZAPATA, I. 2012. Evaluation of native strains of Isaria fumosorosea (Wize) against Anastrepha ludens (Loew) (Diptera: Tephritidae). Journal of Life Science 6 (11): 957-960.

GARCÍA, A. S.; MESSIAS, C. L.; SOUZA H. M. L.; PIEDRABUENA, A. E. 1984. Patogenicidade de Metarhizium anisopliae var. anisopliae a Ceratitis capitata (Wiedemann) (Diptera: Tephritidae). Revista Brasileira de Entomologia 28 (4): 421-424.

GAZIT, Y.; ROSSLER, Y.; WANG, S.; TANG, J.; LURIE, S. 2004. Commodity treatment and quarantine entomology. Thermal death kinetics of egg and third instar Mediterranean fruit fly (Diptera: Tephritidae). Journal of Economic Entomology 97 (5): 1540-1546.

GUPTA, S.; KRASNOFF, S. B. 1991. Isolation of beauvericin as an insect toxin from Fusarium semitectum and Fusarium moniliforme var. subglutinans. Mycopathologia 115 (3): 185-189.

HERNÁNDEZ DÍAZ-ORDAZ, N.; PÉREZ, N.; TOLEDO, J. 2010. Patogenicidad de tres cepas de hongos entomopatógenos a adultos de Anastrepha obliqua (Macquart) (Diptera: Tephritidae) en 
condiciones de laboratorio. Acta Zoológica Mexicana 26 (3): 481-494.

HERNÁNDEZ-ORTIZ, V. 2007. Diversidad y biogeografía del género Anastrepha en México. pp. 53-76. En: Hernández-Ortiz, V. (Ed.). Moscas de la fruta en Latinoamérica (Diptera, Tephritidae): Diversidad, Biología y Manejo. D. F., México. S y G editors. $167 \mathrm{p}$.

JACKSON, M. A.; MCGUIRE, M. R.; LACEY, L. A.; WRAIGHT, S. P. 1997. Liquid culture production of desiccation tolerant blastospores of the bioinsecticidal fungus Paecilomyces fumosoroseus. Mycological Research 101 (1): 35-41.

JEGOROV, A.; SEDMERA, P.; MATHA, V.; SIMEK, P.; ZAHRADNÍČKOVÁ, H.; LANDA, Z.; EYAL, J. 1994. Beauverolides $\mathrm{L}$ and La from Beauveria tenella and Paecilomyces fumosoroseus. Phytochemistry 37 (5): 1301-1303.

LACEY, L. A.; KIRK, A. A.; MILLAR, L.; MERCADIER, G.; VIDAL, C. 1999. Ovicidal and larvicidal activity of conidia and blastospores of Paecilomyces fumosoroseus (Deuteromycotina: Hyphomycetes) against Bemisia argentifolii (Homoptera: Aleyrodidae) with a description of a bioassay system allowing prolonged survival of control insects. Biocontrol Science and Technology 9 (1): 9-18.

LACEY, L. A.; WRAIGHT, S. P.; KIRK, A. A. 2008. Entomopathogenic fungi for control of Bemisia spp.: Foreign exploration, research and implementation. pp. 33-69. En: Gould, J. K.; Hoelmer, K.; Goolsby, J. (Eds.). Classical biological control of Bemisia tabaci in the USA: a review of interagency research and implementation, volumen 4 of Progress in Biological Control. Springer Science \& Business Media. 361 p.

LANE, B. S.; TRINCI, A. P. J.; GILLESPIE, A. T. 1991. Influence of cultural conditions on the virulence of conidia and blastospores of Beauveria bassiana to the green leafhopper, Nephotettix virescens. Mycological Research 95 (7): 829-833.

LECUONA, R.; PAPIEROK, B.; RIBA, G. 1996. Hongos entomopatógenos. pp. 35-60. En: Lecuona, R. E. (Ed.). Microorganismos patógenos empleados en el control microbiano de insectos plaga. Buenos Aires, Argentina, 338 p.

LEZAMA-GUTIÉRREZ, R.; TRUJILLO- DE LA LUZ, A.; MOLINA-OCHOA, J.; REBOLLEDO-DOMÍNGUEZ, O.; PESCADOR, A. R.; LÓPEZ-EDWARDS, M.; ALUJA, M. 2000. Virulence of Metarhizium anisopliae (Deuteromycotina: Hyphomycetes) on Anastrepha ludens (Diptera: Tephritidae): Laboratory and field trials. Journal of Economic Entomology 93 (4): 1080-1084.

MUÑOZ, J. A.; DE LA ROSA, W.; TOLEDO, J. 2009. Mortalidad en Ceratitis capitata (Wiedemann) (Diptera: Tephritidae) por diversas cepas de Beauveria bassiana (Bals.) Vuillemin, en condiciones de laboratorio. Acta Zoológica Mexicana 25 (3): 609-624.
OVCHNINNIKOV, Y. A.; IVANOV, V. T.; MIKHALEVA, L. L. 1971.The synthesis and some properties of beauvericine. Tetrahedron Letters 12 (2): 159-162.

QUESADA-MORAGA, E.; RUIZ-GARCÍA, A.; SANTIAGO-ÁLVAREZ, C. 2006. Laboratory evaluation of entomopathogenic fungi Beauveria bassiana and Metarhizium anisopliae against puparia and adults of Ceratitis capitata (Diptera: Tephritidae). Journal of Economic Entomology 99 (6): 1955-1966.

ROBERTS, D. W. 1981. Toxins of entomopathogenic fungi. pp. 441- 464. En: Burges, H. D. (Ed.). Microbial control of pests and plant diseases 1970-1980. Londres, Reino Unido, Academic Press. 949 p.

ROBERTS, D. W. 1989. World picture of biological control of insects by fungi. Memorias do. Instituto Oswaldo Cruz 84 (supl. 3): $89-100$.

STIBICK, J. N. L. 2004. General reference for fruit fly programs Tephritidae. USDA, APHIS, PPQ, Pest Detection and Management Programs. Disponible en: https://www.aphis.usda.gov/ import export/plants/manuals/domestic/downloads/economic fruit_fly.pdf. [Fecha revisión: abril 2016].

WATANABE, H. 1987. The host population. pp. 71-112. En: Fuxa, J. R.; Tanada, Y. (Eds.). Epizootiology of insect diseases. N.Y., EE. UU. John Wiley \& Sons. 555 p.

WEEMS, H. V. JR.; HEPPNER, J. B.; STECK, G. J.; FASULO, T. R.; NATION,J. L. 2001. Mexican fruit fly Anastrepha ludens (Loew) (Diptera: Tephritidae). Entomology Circular No. 16. EENY-201. Florida Department of Agriculture and Consumer Services, Division of Plant Industry. 5 p.

YEE, W. L.; LACEY, L. A. 2005. Mortality of different life stages of Rhagoletis indifferens (Diptera: Tephritidae) exposed to the entomopathogenic fungus Metarhizium anisopliae. Journal Entomological Science 40 (2): 167-177.

ZIMMERMANN, G. 2008. The entomopathogenic fungi Isaria farinosa (formerly Paecilomyces farinosus) and the Isaria fumosorosea species complex (formerly Paecilomyces fumosoroseus): biology, ecology and use in biological control. Biocontrol Science and Technology 18 (9): 865-901.

Recibido: 24-sep-2016 • Aceptado: 3-feb-2018

Citación sugerida:

GANDARILLA-PACHECO, F. L.; ELÍAS-SANTOS, M.; FLORES-GONZÁLEZ, M. S.; DE LUNA-SANTILLANA, E. J.; QUINTERO-ZAPATA, I. 2018. Virulencia de blastosporas de Isaria fumosorosea nativas del noreste de México sobre Anastrepha ludens (Diptera: Tephritidae). Revista Colombiana de Entomología 44 (2): 187-192. Julio-Diciembre 2018. 